\title{
Malignant Breast Eccrine Spiradenoma
}

National Cancer Institute

\section{Source}

National Cancer Institute. Malignant Breast Eccrine Spiradenoma. NCI Thesaurus. Code C5180.

A rare tumor characterized by malignant transformation of an eccrine spiradenoma of the breast. 